\title{
ON THE DEVELOPMENT OF FUNCTIONS IN SERIES OF ORTHOGONAL POLYNOMIALS*
}

\author{
BY J. A. SHOHAT
}

Introduction. A function $p(x)$, called the weight or characteristic function, non-negative on a given interval $(a, b)$, finite or infinite, and such that all moments $\dagger$

$$
\alpha_{n}=\int p(x) x^{n} d x, \quad(n=0,1,2, \cdots),
$$

exist, with $\alpha_{0}>0$, gives rise, as is known [1], $\ddagger$ to a system of orthogonal and normal Tchebycheff polynomials $(O P)$

(2) $\phi_{n}(x ; p) \equiv \phi_{n}(x)=a_{n} x^{n}+\cdots, \quad\left(n=0,1, \cdots ; a_{n}>0\right)$,

uniquely determined by the following relations:

$$
\int p(x) \phi_{m}(x) \phi_{n}(x) d x=\delta_{m n}= \begin{cases}0, & m \neq n, \\ 1, & m=n,\end{cases}
$$

$(m, n=0,1, \cdots)$. If $G_{s}(x)=\sum_{i=0}^{s} g_{i} x^{i}$ denotes an arbitrary polynomial of degree $\leqq s$ (subject in some cases to certain explicitly stated conditions), (2) yields

$$
\int p(x) \phi_{n}(x) G_{n-1}(x) d x=0, \quad(n=1,2, \cdots) .
$$

The most important and best known $O P$ are the so-called "classical" polynomials of

(J) Jacobi: $\quad(a, b)$ finite, say, $(-1,1)$;

$$
p(x)=(1+x)^{\alpha-1}(1-x)^{\beta-1},(\alpha, \beta>0) .
$$

(5) (L) Laguerre: $(a, b)=(0, \infty) ; p(x)=x^{\alpha-1} e^{-x}, \quad(\alpha>0)$.

(H) Hermite: $(a, b)=(-\infty, \infty) ; p(x)=e^{-x^{2}}$.

We note the following important cases of $(\mathrm{J})$ :

* Address delivered by invitation of the Program Committee, at the Summer Meeting of the Society, September 6, 1934.

$\dagger$ Here and hereafter $\int$ means $\int_{a}^{b}$.

$\$$ Numbers in brackets refer to the Bibliography at the end. 
Legendre polynomials $(\alpha=\beta=0)$, trigonometric polynomials $(\alpha=\beta=1 / 2)$.

The $O P$ have many interesting and increasingly important applications in pure analysis, as well as in mathematical physics, mathematical statistics, and mechanics. We may mention approximate evaluation of definite integrals (mechanical quadratures), interpolation, curve fitting, certain oscillation problems in engineering mechanics, and investigation of certain classes of polynomials (for example, monotonic in $(a, b)$ ). The most important of such applications, which served to introduce the general $O P$ in analysis, is their use in expanding arbitrary functions in series. In fact, making use of (3), we get the formal expansion

$$
f(x) \sim \sum_{n=0}^{\infty} f_{n} \phi_{n}(x), \quad f_{n}=\int p(x) f(x) \phi_{n}(x) d x,
$$

of any function $f(x)$, for which the integrals expressing the $f_{n}$ exist. We see that (6) is built up in the same manner as the trigonometric expansion, the ordinary Fourier series. The latter employs the simplest orthogonal sequence $\{\sin n x, \cos n x\}$, $(n=0,1, \cdots)$, while (6) employs orthogonal polynomials, which may be considered the next simplest orthogonal functions.

A fundamental question naturally arising in connection with the expansion (6) is that of its convergence. Given the sequence $\left\{\phi_{n}(x)\right\}$, for what classes of functions $f(x)$ does (6) converge in $(a, b)$ or in a part thereof? The present paper is devoted to a discussion of various methods used in dealing with the convergence properties of the expansion (6). It is confined to a single real variable and to ordinary convergence. The proofs are in general but briefly sketched, if not omitted, our aim being to bring out general ideas. The topics treated can be classified as follows.

I. General properties of the expansion (6).

II. General methods for investigating the convergence of (6).

(a) Case of continuous functions; use of Weierstrass' theorem.

(b) Application of the theory of integral equations.

(c) Application of the general theory of orthogonal functions; Rademacher-Menchoff theorem, Lebesgue constants.

(d) The use of the asymptotic expression of $\phi_{n}(x),(n \rightarrow \infty)$ (Dirichlet integral); equiconvergence. 
III. Special methods for investigating the convergence of (6).

(a) From summability to convergence.

(b) Use of the closure equation.

(c) Use of the differential equation (for the classical $O P$ ).

(d) Comparison method.

These methods naturally vary in generality and power. This is best illustrated by applying the various methods to expansions in series of Legendre polynomials, the oldest and the best known $O P$. A comparison of the criteria of convergence thus obtained brings out the following fact: the more general the method, the less does it utilize the special character of the functions involved; hence, a gain in generality is accompanied, as a rule, by a loss in the preciseness of the results obtained.

The supreme goal, when dealing with (6), is to show that, regarding convergence, it behaves in a certain subinterval of $(a, b)$ like the ordinary Fourier series expansion of $f(x)$ or of some function simply related to it. This constitutes what we call the equiconvergence theorem, of greatest importance in our theory. Indeed, we well know the wide range of validity of the Fourier series expansion, which makes it such a powerful analytical instrument. The classes of $O P$ for which this goal is attainable are evidently the most interesting, and the method by which the goal has been attained is evidently the most powerful one.

I. Some General Properties of the Expansion (6).

1. Various Representations of the Remainder in (6). We rewrite (6) in the form

$$
f(x)=\sum_{i=0}^{n} f_{i} \phi_{i}(x)+R_{n}(x ; f) \equiv S_{n}(x ; f)+R_{n}(x ; f),
$$

whence, by use of (3) and (4), it follows that $R_{n}\left(x ; G_{n}\right) \equiv 0$,

$$
\begin{gathered}
1=\int p(y) \sum_{i=0}^{n} \phi_{i}(x) \phi_{i}(y) d y \equiv \int p(y) K_{n}(x, y) d y, \\
S_{n}(x ; f)=\int p(y) f(y) K_{n}(x, y) d y,
\end{gathered}
$$

and, by use of Darboux's formula [2], 


$$
\begin{aligned}
K_{n}(x, y) & \equiv \sum_{i=0}^{n} \phi_{i}(x) \phi_{i}(y) \\
& \equiv \frac{a_{n}}{a_{n+1}}\left[\frac{\phi_{n+1}(x) \phi_{n}(y)-\phi_{n}(x) \phi_{n+1}(y)}{x-y}\right],
\end{aligned}
$$

$$
\begin{aligned}
K_{n}(x, x) & =K_{n}(x)=\frac{a_{n}}{a_{n+1}}\left[\phi_{n+1}^{\prime}(x) \phi_{n}(x)-\phi_{n}^{\prime}(x) \phi_{n+1}(x)\right] \\
& =\sum_{i=0}^{n} \phi_{i}{ }^{2}(x) .
\end{aligned}
$$

$$
S_{n}(x ; f)=\frac{a_{n}}{a_{n+1}} \int p(y) f(y) \frac{\phi_{n+1}(x) \phi_{n}(y)-\phi_{n}(x) \phi_{n+1}(y)}{x-y} d y,
$$

$$
R_{n}(x) \equiv R_{n}(x ; f)=\int p(y)[f(x)-f(y)] K_{n}(x, y) d y
$$

$$
\begin{gathered}
=\frac{a_{n}}{a_{n+1}} \int p(y)[f(x)-f(y)] \frac{\phi_{n+1}(x) \phi_{n}(y)-\phi_{n}(x) \phi_{n+1}(y)}{x-y} d y \\
R_{n}(x ; f)=F(x)-\int p(y)[F(x)-F(y)] K_{n}(x, y) d y \\
F(x) \equiv f(x)-G_{n}(x)
\end{gathered}
$$

2. Bessel Inequality. Closure. The general assumption will be now made that $f(x)$ is of the class $L_{p}^{2}$, that is, $p(x) f^{2}(x)$ is $L$-integrable in $(a, b)$. (Similarly, $f(x)$ being of the class $L_{p}$ means $p(x) f(x)$ is $L$-integrable.) A ready application of (3) yields

$$
\int p(y)\left[f(y)-\sum_{i=0}^{n} f_{i} \phi_{i}(y)\right]^{2} d y=\int p(y) f^{2}(y) d y-\sum_{i=0}^{n} f_{i}{ }^{2},
$$

which leads to the Bessel inequality:

$$
\begin{aligned}
& \int p(y)\left[f(y)-S_{n}(y ; f)\right]^{2} d y=\int p(y) R_{n}{ }^{2}(y) d y \\
& =\int p(y) f^{2}(y) d y-\sum_{i=0}^{n} f_{i}{ }^{2} \geqq 0, \quad(n=0,1, \cdots) .
\end{aligned}
$$

Hence we may write

$$
\sum_{n=0}^{\infty} f_{n}^{2} \text { converges and is } \leqq \int p(y) f^{2}(y) d y .
$$


If in (14) we have the right to use the equality sign, then we have the so-called Parseval formula (closure equation), one of the most important in the theory of orthogonal functions:

$$
\sum_{n=0}^{\infty} f_{n}^{2}=\int p(y) f^{2}(y) d y,
$$

where $f(x)$ is of class $L_{p}{ }^{2}$, and $f_{n}=\int p(x) f(x) \phi_{n}(x) d x$. This, in connection with (13), leads to the equation

$$
\int p(y) R_{n}^{2}(y) d y=\sum_{i=n+1}^{\infty} f_{i}^{2}=o(1), \quad(n \rightarrow \infty) .
$$

In the theory of $O P$ it is shown: (i) Parseval's formula always holds for $(a, b)$ finite; (ii) for $(a, b)$ infinite its validity is intimately connected with the character, determined or indeterminate, of the moment-problem related to $p(x)$, that is, the problem whether the system of infinitely many equations

$$
\int x^{n} d \psi(x)=\int p(x) x^{n} d x=\alpha_{n}, \quad(n=0,1, \cdots),
$$

where the unknown function $\psi(x)$ is monotonic non-decreasing in $(a, b)$ with $\psi(a)=0$ (and the left-hand integral is a Stieltjes integral), has or has not solutions distinct from the given one: $\psi(x)=\int_{a}^{x} p(x) d x . *$ In particular, Parseval's formula holds for the polynomials of Laguerre and Hermite. The validity of Parseval's formula is assumed throughout the subsequent discussion.

3. Consequences Derived from Parseval's Formula. We know that

$$
\lim _{n \rightarrow \infty} f_{n}=0,
$$

for any $f(x)$ of the class $L_{p}^{2}$. The set of relations

$$
\int p(x) f(x) \phi_{n}(x) d x=0, \quad(n=0,1, \cdots),
$$

implies $(p(x))^{1 / 2} f(x)=0$ almost everywhere in $(a, b) . \dagger$ In other words, two functions having the same Fourier coefficients

* The sharp distinction between the cases of $(a, b)$ finite and infinite, as stated above, is clearly seen from the following example due to Stieltjes [3]: $\int_{0}^{\infty} e^{-x^{1 / 4}} \sin \left(x^{1 / 4}\right) x^{n} d x=0, \quad(n=0,1,2, \cdots)$.

$\dagger$ If $(a, b)$ is finite, $(18)$ is valid for any $f(x)$ of the class $L_{p}$ in $(a, b)$. In fact, 
in (6) are equivalent, that is, their difference multiplied by $(p(x))^{1 / 2}$ vanishes almost everywhere in $(a, b) .^{*}$

The product

$$
(p(x))^{1 / 2} S_{n}(x ; f)
$$

converges, for $n \rightarrow \infty$, on the average to $(p(x))^{1 / 2} f(x)$ on $(a, b)$ [4]. It follows that a subsequence $(p(x))^{1 / 2} S_{n_{k}}(x ; f)$ can be extracted which converges to $(p(x))^{1 / 2} f(x)$ in the ordinary sense $\left(n_{k} \rightarrow \infty\right)$ almost everywhere in $(a, b)$. Hence, if $S_{n}(x ; f)$ converges, for $n \rightarrow \infty$, on a set $E \subset(a, b)$ of positive measure (where we assume $p(x) \neq 0)$, it necessarily converges to the value $f(x)$ almost everywhere on $E$.

We have also

$$
\int p(x) f(x) F(x) d x=\sum_{n=0}^{\infty} f_{n} F_{n}
$$

where $F$ is of class $L_{p}{ }^{2}$, and

$$
F_{n}=\int p(x) F(x) \phi_{n}(x) d x .
$$

This is readily obtained by applying (15) to $f(x) \pm F(x)$. Assume further that

$$
\int_{c}^{d} \frac{d x}{p(x)} \text { exists, } \quad(c, d) \subset(a, b) .
$$

Take in (20), $F(x)=1 / p(x)$ in $(c, d)$, and $F(x)=0$ elsewhere in $(a, b)$; then $F_{n}=\int_{c}^{d} \phi_{n}(x) d x$, and we thus obtain the following important result:

$$
\int_{c}^{d} f(x) d x=\sum_{n=0}^{\infty} f_{n} \int_{c}^{d} \phi_{n}(x) d x,
$$

which tells us that the expansion (6), whether convergent or not, can be integrated term by term in any interval $(c, d)$ which is part

reduce $(a, b)$ to $(-1,1)$. Put $x=\cos \theta$ and denote $p(\cos \theta) f(\cos \theta)$ by $F(\theta)$. Then $\int_{1}^{l} p(x) f(x) \phi_{n}(x) d x=0,(n=0,1, \cdots)$, is equivalent to $\int_{-1}^{1} p(x) f(x) x^{n} d x$ $=0$ or to $\int_{0}{ }^{\pi} F(\theta) \cos n \theta d \theta=0,(n=0,1, \cdots)$. Define $F_{1}(\theta)=F(\theta)$ in $(0, \pi)$, and $F_{1}(\theta)=-F(-\theta)$ in $(-\pi, 0)$. Then $\int_{-\pi}^{\pi} F_{1}(\theta) \underset{\sin }{\cos } n \theta d \theta=0$ for all $n \geqq 0$; hence, by the theory of trigonometric series, $F_{1}(\theta)=0$ almost everywhere in $(-\pi, \pi)$.

* Stieljes [3]. 
of $(a, b)$, where (21) is satisfied, ${ }^{*}$ the convergence in (22) being uniform if $d$ varies.

We now turn again to the remainder $R_{n}(x ; f)$ in $(6)$ and assume, for definiteness, $x$ to be a fixed point inside $(a, b)$. Rewrite (11) as follows:

$$
\begin{array}{r}
R_{n}(x ; f)=\frac{a_{n}}{a_{n+1}}\left(\int_{a}^{x-\epsilon}+\int_{x-\epsilon}^{x+\epsilon}+\int_{x+\epsilon}^{b}\right) p(y)[f(x)-f(y)] \\
\cdot K_{n}(x, y) d y \equiv i_{1}+i_{2}+i_{3} \\
=\frac{a_{n}}{a_{n+1}}\left\{\phi_{n+1}(x) \int_{a}^{x-\epsilon} p(y) \frac{f(x)-f(y)}{x-y} \phi_{n}(y) d y\right. \\
\left.-\phi_{n}(x) \int_{a}^{x-\epsilon} p(y) \frac{f(x)-f(y)}{x-y} \phi_{n+1}(y) d y\right\}+i_{2}+i_{3} .
\end{array}
$$

(Here and hereafter $\epsilon$ denotes a sufficiently small positive quantity, properly chosen.) In $i_{1}$ (similar considerations apply to $i_{3}$ ) define

Then

$$
\psi(y)=\frac{f(x)-f(y)}{x-y} \text { in }(a, x-\epsilon), \quad \psi(y)=0 \text { in }(x+\epsilon, b):
$$

$$
\int_{a}^{x-\epsilon} p(y) \frac{f(x)-f(y)}{x-y} \phi_{n}(y) d y=\int_{a}^{b} p(y) \psi(y) \phi_{n}(y) d y,
$$

and we thus conclude, by (17), that

$$
\lim _{n \rightarrow \infty} \int_{a}^{x-\epsilon} p(y) \frac{f(x)-f(y)}{x-y} \phi_{n}(y) d y=0 .
$$

Furthermore, if $(a, b)$ is finite, then $a_{n} / a_{n+1}$ is bounded [1]: $a_{n} / a_{n+1}=O(1)$. If, in addition,

$$
\phi_{n}(x)=O(1)
$$

at the given point $x$, then

$$
\lim _{n \rightarrow \infty} \frac{a_{n}}{a_{n+1}} \phi_{n+1}(x) \int_{a}^{x-\epsilon} p(y) \frac{f(x)-f(y)}{x-y} \phi_{n}(y) d y=0 .
$$

It follows that the behavior of $R_{n}(x ; f)$ depends here solely

* For Legendre polynomials (22) holds for any $f(x)$ of the class $L[5]$. 
upon its component $i_{2}$. The important conclusion resulting from the above considerations can be stated as follows. For $(a, b)$ finite, the convergence of the expansion (6) to $f(x)$ at a given point $x$ where the sequence $\left\{\phi_{n}(x)\right\}$ remains bounded for $n \rightarrow \infty$ depends solely upon the nature of $f(x)$ in the immediate neighborhood of $x$ [6].

In other words, if two functions $f_{1}(x)$ and $f_{2}(x)$ coincide in an arbitrarily small neighborhood $(x-\epsilon, x+\epsilon)$ of the point $x$, then

$$
\begin{aligned}
& \lim \left[R_{n}\left(x ; f_{1}\right)-R_{n}\left(x ; f_{2}\right)\right]=0, \\
& \lim \left[S_{n}\left(x ; f_{1}\right)-S_{n}\left(x ; f_{2}\right)\right]=0, \quad(n \rightarrow \infty),
\end{aligned}
$$

for $R_{n}\left(x ; f_{1}-f_{2}\right)$ is here reduced to the components $i_{1}$ and $i_{3}$ in (23), written for $f_{1}(x)-f_{2}(x)$.

We now add the further condition that $f(x)$ satisfies a Lipschitz condition in the neighborhood of the point $x$, that is,

$$
\left|f\left(y^{\prime}\right)-f\left(y^{\prime \prime}\right)\right| \leqq \lambda\left|y^{\prime}-y^{\prime \prime}\right|
$$

$\left(x-\epsilon \leqq y^{\prime}, y^{\prime \prime} \leqq x+\epsilon ; \lambda=\right.$ const., independent of $\left.y^{\prime}, y^{\prime \prime}\right)$.

Define $F(y)$ as follows:

$$
\begin{aligned}
F(y) & =\frac{f(x)-f(y)}{x-y} \text { in }(x-\epsilon, x+\epsilon), y \neq x, \\
& =0 \text { elsewhere in }(a, b), \text { and for } y=x .
\end{aligned}
$$

Under condition (28), $F(y)$ is seen to be of the class $L_{p}{ }^{2}$, and by the same reasoning as applied above to $i_{1,3}$, we show that here $\lim i_{2}=0$, so that $\lim R_{n}(x ; f)=0,(n \rightarrow \infty)$. The same conclusion holds if

$$
\left|f\left(y^{\prime}\right)-f\left(y^{\prime \prime}\right)\right| \leqq \lambda\left|y^{\prime}-y^{\prime \prime}\right|^{\alpha} \text {, with } \alpha>1 / 2,
$$

where $\lambda, y^{\prime}, y^{\prime \prime}$ are as in (28) (Lipschitz condition of order $\alpha$ ), provided $p(x)$ is bounded in $(x-\epsilon, x+\epsilon)$. Thus we have found, as an immediate consequence of Parseval's formula, that the expansion (6) converges to $f(x)$ at any point $x$ in the finite interval $(a, b)$ in the neighborhood of which $f(x)$ satisfies a Lipschitz condition of order 1 (or of order $>1 / 2$, if $p(x)$ is bounded in this neighborhood), provided the sequence $\left\{\phi_{n}(x)\right\}$ is bounded at the point $x$ under consideration.

The latter condition is satisfied for various classes of $O P$, often uniformly in $(a+\epsilon, b-\epsilon)$, as illustrated by Jacobi poly- 
nomials. (This is usually established by means of the asymptotic expression for $\phi_{n}(x)$.)

4. Some Extremal Properties of the Expansion (6). By means of (3) and (4) we readily derive the following relations:

$$
\begin{aligned}
\int p(y)\left[f(y)-\sum_{i=0}^{n}\left(f_{i}+h_{i}\right) \phi_{i}(y)\right]^{2} d y \\
=\int p(y) f^{2}(y)-\sum_{i=0}^{n} f_{i}{ }^{2}+\sum_{i=0}^{n} h_{i}{ }^{2},
\end{aligned}
$$

where the $h_{i}$ denote arbitrary constants; whence, by (13),

$$
\int p(y)\left[f(y)-S_{n}(y ; f)\right]^{2} d y \leqq \int p(y)\left[f(y)-G_{n}(y)\right]^{2} d y
$$

where the equality sign holds only for $G_{n}(y) \equiv S_{n}(y ; f)$. This is an important extremal property which led Tchebycheff to the introduction of the general $O P$ in analysis, considering (6) as furnishing interpolation in the sense of least squares.

\section{General Methods for Investigating the Convergence Properties OF THE Expansion (6).}

1. Case of Continuous Functions. The convergence of (6) evidently depends upon the order of magnitude (with respect to $n$ ) of the chosen polynomials $\phi_{n}(x)$ and of the coefficient $f_{n}$. While the former may be investigated once for all, by studying the properties of $p(x)$, the latter essentially depends on the nature of $f(x)$. We proceed to show that the study of the order of $f_{n}$ is readily achieved in the important case where $f(x)$ is continuous in the finite interval $(a, b)$. Here one naturally thinks first of Weierstrass' approximation theorem: any function continuous in a given finite interval can be therein approximated uniformly and indefinitely by means of polynomials of ever increasing degrees. Moreover, Tchebycheff has shown, that among all polynomials of degree $\leqq n$, there exists a unique polynomial $\Pi_{n}(x ; f)$ of "best approximation" $\left(=E_{n}(f)\right)$ to $f(x)$ on $(a, b)$, that is,

$$
E_{n}(f)=\max \left|f(x)-\Pi_{n}(x ; f)\right| \leqq \max \left|f(x)-G_{n}(x)\right|,
$$

$(a \leqq x \leqq b)$, where, by Weierstrass' theorem,

$$
\lim _{n \rightarrow \infty} E_{n}(f)=0 \text {. }
$$


Here, as in many other problems dealing with continuous functions, Weierstrass' theorem proves a powerful and readily applicable tool.

(i) We have, first, by (4),

$$
\begin{aligned}
f_{n} & =\int p(y)\left[f(x)-G_{n-1}(x)\right] d x \\
& =\int p(x)\left[f(x)-\Pi_{n-1}(x ; f)\right] \phi_{n}(x) d x .
\end{aligned}
$$

Hence, applying Schwartz's inequality and making use of (31), we find that

$$
\left|f_{n} \phi_{n}(x)\right| \leqq\left(\alpha_{0}\right)^{1 / 2} E_{n-1}(f)\left|\phi_{n}(x)\right| .
$$

While this estimate, resulting from very simple considerations, is rather crude, it has the advantage of showing in many cases the convergence of (6) directly, without any further discussion. In fact, the order of magnitude of $E_{n}(f)$, depending upon the continuity properties of $f(x)$, is well known, thanks to the work of Lebesgue, de la Vallée-Poussin, S. Bernstein, and Dunham Jackson. Thus

$$
\left|f^{(p)}\left(x^{\prime}\right)-f^{(p)}\left(x^{\prime \prime}\right)\right| \leqq \lambda\left|x^{\prime}-x^{\prime \prime}\right|^{\alpha}, \quad\left(a \leqq x^{\prime}, x^{\prime \prime} \leqq b ; 0<\alpha \leqq 1\right),
$$

implies $E_{n}(f)=O\left(n^{-p-\alpha}\right),(p \geqq 0)$;

$$
\begin{aligned}
& f^{(p)}(x) \text { continuous in }(a, b) \text { implies } E_{n}(f)=o\left(n^{-p}\right) ; \\
& |f(x+\delta)-f(x)| \cdot|\log | \delta||_{\delta \rightarrow 0} \rightarrow 0 \text { (Dini-Lipschitz condition) }
\end{aligned}
$$

implies $E_{n}(f)=o(1 / \log n)$.

If now the order of $\phi_{n}(x)$ is known, say

$$
\left|\phi_{n}(x)\right|=O\left(n^{\sigma}\right)
$$

for a certain $x$, then, for the same $x$,

$$
\left|f_{n} \phi_{n}(x)\right|=O\left(n^{\sigma} E_{n-1}(f)\right) .
$$

This, combined with (35), enables us to indicate at once classes of continuous functions for which (6) converges for the above $x$. If, for example, $\sigma=0,1$, then (6) certainly converges (absolutely) for the above values of $x$, if the first or the second 
derivative of $f(x)$, respectively, satisfies in $(a, b)$ a Lipschitz condition of an arbitrary positive order $\alpha$, for then

$$
\left|f_{n} \phi_{n}(x)\right|=O\left(n^{-1-\alpha}\right), \quad(\alpha>0) .
$$

The said convergence is uniform in any interval $\subset(a, b)$ in which $\left|\phi_{n}(x)\right|=O\left(n^{\sigma}\right)$, with $\sigma=0,1$.

(ii) More refined results are obtained from (12), with $G_{n}(x) \equiv \Pi_{n}(x ; f)$. We thus get, applying Schwartz's inequality to the integral on the right, and using (3) once more,

$$
\begin{aligned}
& \left|R_{n}(x ; f)\right| \leqq E_{n}(f)\left\{1+\left(K_{n}(x)\right)^{1 / 2}\right\}=O\left(E_{n}(f)\left(K_{n}(x)\right)^{1 / 2}\right) \\
& \left|R_{n}(x ; f)\right|=O\left(E_{n}(f) n^{\sigma+1 / 2}\right), \text { if }\left|\phi_{n}(x)\right|=O\left(n^{\sigma}\right)
\end{aligned}
$$

where $\sigma>-1$. We recall that $a_{n} / a_{n+1}=O(1)$ for $(a, b)$ finite.

(iii) Still more refined results can be obtained $[7,8]$, if we again use (12), breaking up the integral on the right somewhat along the lines of (23):

$$
\begin{aligned}
\int_{a}^{b} & =\int_{a}^{c+\epsilon}+\int_{c+\epsilon}^{x-\epsilon_{n}}+\int_{x-\epsilon_{n}}^{x+\epsilon_{n}}+\int_{x+\epsilon n}^{x+\epsilon}+\int_{x+\epsilon}^{b} \\
& \equiv i_{1}+i_{2}+i_{3}+i_{4}+i_{5}, \\
(a<c \leqq & \left.\leqq d<b ; \epsilon_{n}=o(1)(n \rightarrow \infty),\left|\phi_{n}(x)\right|=O\left(n^{\sigma}\right), \sigma>-1\right) .
\end{aligned}
$$

In $i_{1,5}$, where $|x-y| \geqq \epsilon$, we use Darboux's formula (9) for $K_{n}(x, y)$ and get integrals of the type $\int p(y)\left|\phi_{n}(y)\right| d y$ which $=O(1)$ (by Schwartz's inequality), so that

$$
\left|i_{1,5}\right|=O\left(n^{\sigma} E_{n}(f)\right) \text {. }
$$

In $i_{2,4}$ put $x-y=u$; then

$$
\left|i_{2,4}\right|=O\left(n^{2 \sigma} E_{n}(f)\right) \int_{\epsilon_{n}}^{b-a} \frac{d u}{u}=O\left(E_{n}(f) \cdot n^{2 \sigma}|\log | \epsilon_{n}||\right) .
$$

Finally, in $i_{3}$ use $K_{n}(y)=O\left(n^{2 \sigma+1}\right)$, so that

$$
\left|i_{3}\right|=O\left(E_{n}(f) \cdot n^{2 \sigma+1} \int_{x-\epsilon_{n}}^{x+\epsilon_{n}} p(y) d y\right) .
$$

By taking $\epsilon_{n}=n^{-\beta}$, with a properly chosen $\beta>0$, we can find the order of $R_{n}(x ; f)$ and the class of continuous functions for which $\lim _{n \rightarrow \infty} R_{n}(x ; f)=0$.

Note. In the case under discussion, by (30) and (16), 
(40) $\int p(y) R_{n}{ }^{2}(y) d y \leqq \int p(y)\left|f(y)-\Pi_{n}(y ; f)\right|^{2} d y \leqq \alpha_{0} E_{n}{ }^{2}(f)$,

$$
E_{n}(f) \geqq \frac{1}{\alpha_{0}^{1 / 2}}\left(\sum_{i=n+1}^{\infty} f_{i}^{2}\right)^{1 / 2} .
$$

A lower bound for $E_{n}(f)$ is of importance in the theory of approximation. The relations (40) and (41), for a suitable orthogonal system $\left\{\phi_{n}(x)\right\}$, furnish such a lower bound, very acceptable for certain classes of functions [9].

2. Application of the Theory of Integral Equations. Rewrite (3) and (6), respectively, as

where

$$
\begin{aligned}
\int \Phi_{m}(x) \Phi_{n}(x) d x & =\delta_{m n}, \quad(m, n=0,1, \cdots), \\
F(x) & \sim \sum_{n=0}^{\infty} f_{n} \Phi_{n}(x),
\end{aligned}
$$

$$
\Phi_{n}(x)=(p(x))^{1 / 2} \phi_{n}(x) ; F(x)=f(x)(p(x))^{1 / 2} ; f_{n}=\int F(x) \Phi_{n}(x) d x .
$$

Consider the formal expression

$$
K(x, y) \equiv \sum_{n=0}^{\infty} \frac{\Phi_{n}(x) \Phi_{n}(y)}{l_{n}}=(p(x) p(y))^{1 / 2} \sum_{n=0}^{\infty} \frac{\phi_{n}(x) \phi_{n}(y)}{l_{n}},
$$

where $l_{n}$ denote constants. For finite $(a, b)$ the positive constants $l_{n}$ in (44) may be chosen in infinitely many ways so that its right-hand member converges absolutely and uniformly in $(a, b)$. Take, for example,

$$
l_{n}=M_{n}^{2} n^{1+\alpha},(\alpha>0), \quad M_{n}=\max \left|\phi_{n}(x)\right| \text { in }(a, b) .
$$

The same holds for $(a, b)$ infinite, if $(p(x))^{1 / 2}\left|\phi_{n}(x)\right|$ does not exceed in $(a, b)$ a finite positive quantity $M_{n}$ for each $n$ (example: polynomials of Laguerre and Hermite). We have then, by virtue of orthogonality,

$$
\begin{aligned}
(p(x))^{1 / 2} \phi_{n}(x) & =l_{n} \int(p(y))^{1 / 2} K(x, y) \phi_{n}(y) d y \\
\Phi_{n}(x) & =l_{n} \int K(x, y) \Phi_{n}(y) d y, \quad(n=0,1, \cdots) .
\end{aligned}
$$


Thus, in cases under consideration, the functions $\left\{\Phi_{n}(x)\right\}$ are the fundamental functions for the linear integral equation (46), and the expansion (43), which is but a modification of (6), may be studied by applying the general theory of linear integral equations, more precisely, the Hilbert-Schmidt expansion theorem, which in its simplest form can be stated as follows. Let $K(x, y)$ be a symmetric continuous kernel in the domain $a \leqq x$, $y \leqq b$, and let $\omega_{n}(x),(n=1,2, \cdots)$, denote the corresponding fundamental functions. Any function of the form

$$
f(x)=\int K(x, y) h(y) d y,
$$

where $h(x)$ is square-integrable in $(a, b)$, can be expanded in a series according to the $\omega_{n}(x)$, which converges absolutely and uniformly; the expansion is of the form

$$
f(x)=\sum_{n=1}^{\infty} \frac{h_{n}}{l_{n}} \omega_{n}(x),
$$

where

$$
h_{n}=\int h(y) \omega_{n}(y) d y,
$$

and where $l_{n}$ denote characteristic numbers.

However, we encounter here two serious difficulties. First, the actual construction of the kernel $K(x, y)$ in (45) and (46), depending largely upon the choice of the constants $l_{n}$ in (44), is not so easy to carry out even in the simplest cases of Legendre and Hermite polynomials [10,11, 12]; secondly, the criteria for convergence of (43) (or (6)) thus obtained are far too strict, for the method, due to its very generality, does not utilize sufficiently the individual properties of the orthogonal functions involved. Thus Weyl [11], applying the theory of integral equations to the expansion of a given function $f(x)$ according to Hermite functions $\left\{e^{-x^{2} / 4} \phi_{n}(x)\right\}$ orthogonal in $(-\infty, \infty)$, finds the said expansion converges if $f(x)$ and $f^{\prime}(x)$ are continuous and both integrals $\int_{-\infty}^{\infty} x^{2} f^{2}(x) d x, \int_{-\infty}^{\infty} f^{\prime 2}(x) d x$ exist. These criteria are far inferior to those obtained by other methods $[13,14]$. Even more stringent are the criteria of W. Lebedeff [12] obtained by means of a different kernel. The same remark 
applies to the discussion by the said writers of expansions in series of Laguerre polynomials.

A different application of the theory of integral equations to $O P$ has been given by $\mathrm{N}$. Kryloff [15]. His main point is the following generalized Schmidt's Lemma.

Consider a sequence $\left\{\omega_{n}(x)\right\},(n=0,1, \cdots)$, of class $L^{2}$, orthogonal and normal in $(a, b)$, for which Parseval's formula holds, that is, for any $f(x)$ of class $L^{2}$,

$$
\int f^{2}(x) d x=\sum_{n=0}^{\infty} f_{n}^{2}, \quad f_{n}=\int f(x) \omega_{n}(x) d x .
$$

If $\psi(x)$ is of class $L^{2}$ and $F(x, z)$ is such that $\int F^{2}(x, z) d z \leqq M$ (independent of $z$ ) for any $z$ in $\left(z_{0}, z_{1}\right)$, then

(49) $\int F(x, z) \psi(x) d x=\sum_{n=0}^{\infty} \int F(x, z) \omega_{n}(x) d x \cdot \int \psi(x) \omega_{n}(x) d x$,

and the right-hand member converges absolutely and uniformly for $z_{0} \leqq z \leqq z_{1}$. (See the Hilbert-Schmidt theorem given above.)

Suppose now we wish to investigate the convergence of the expansion

$$
f(x)=\sum_{n=0}^{\infty} f_{n} \phi_{n}(x), \quad f_{n}=\int p(x) f(x) \phi_{n}(x) d x .
$$

The ingenious idea of Kryloff is to identify (6) with (49), and then to apply the preceding Lemma. Choose, first, $F(x, z)$ and the sequence $\left\{\omega_{n}(x)\right\}$, then $\psi(x)$, so that

$$
\begin{aligned}
\int F(x, z) \omega_{n}(x) d x & =\phi_{n}(z), & (a \leqq z \leqq b), \\
\int \psi(x) \omega_{n}(x) d x & =\int p(x) f(x) \phi_{n}(x) d x, & (n=0,1, \cdots) .
\end{aligned}
$$

If such a choice is possible, the right-hand member in (6) (where $x$ is replaced by $z$ ) does become identical with that in (49), namely,

$$
\sum_{n=0}^{\infty} f_{n} \phi_{n}(z) \equiv \sum_{n=0}^{\infty} \int \psi(x) \omega_{n}(x) d x \cdot \int F(x, z) \omega_{n}(x) d x .
$$

Hence it converges absolutely and uniformly in $(a, b)$ to the value of $f(x)$ (by virtue of (19)), if $F(x, z)$ satisfies the condition 
of the Lemma. Kryloff applies this method to Jacobi polynomials in $(-1,1)$ and shows, on the basis of their differential equation (see below, (85)), that such a choice as required by (50) is possible, if $\alpha, \beta>1$, and the expansion (6) converges absolutely and uniformly in $(-1,1)$, if $f(x)$ and $f^{\prime}(x)$ are of class $L_{p}^{2}$.

3. Application of the General Theory of Orthogonal Functions. This is suggested by the very form of the expansion (43). The application in question, in order to attain the utmost in effectiveness, should be coupled with the special properties of the $O P$ involved.

(i) Use of Rademacher-Menchoff theorem [16, 17]. This general theorem deals with a sequence $\left\{\omega_{n}(x)\right\}$ of functions orthogonal and normal in the finite interval $(a, b)$, and tells us that the expansion $\sum_{n=0}^{\infty} c_{n} \omega_{n}(x)$ converges almost everywhere in $(a, b)$, if $\sum_{n=2}^{\infty} c_{n}^{2} \log ^{2} n$ converges. ${ }^{*}\left(\omega_{n}(x)\right.$ is of class $L^{2}$.)

This theorem, applied to (43), shows at once that (43) converges to $(p(x))^{1 / 2} f(x)$ almost everywhere in the finite interval $(a, b)$, if the coefficients $f_{n}$ are such that $\sum_{n=2}^{\infty} f_{n}^{2} \log ^{2} n$ converges. The latter condition is satisfied in one of the following cases:

1. $0<p(x)<M$, ( $M$ finite), or $0<p(x)<M /((x-a)(b-x))^{1 / 2}$, $f(x)$ is of bounded variation in $(a, b)[18]$.

2. $p(x)>0 ;\left|f\left(x^{\prime}\right)-f\left(x^{\prime \prime}\right)\right|<$ const. $/|\log | x^{\prime}-x^{\prime \prime}||^{1+\epsilon},\left(a \leqq x^{\prime}\right.$, $\left.x^{\prime \prime} \leqq b\right) \dagger[19]$. The Rademacher-Menchoff theorem lays the emphasis on the coefficients $f_{n}$ in the expansion (6).

(ii) Use of Lebesgue constants. Here the emphasis is placed on the orthogonal functions employed. Using the expressions (11) and (12) for the remainder $R_{n}(x ; f)$ in the expansion (6), we are led to introduce the Lebesgue constants:

$$
\begin{aligned}
& \rho_{n}(x)=\int p(y)\left|K_{n}(x, y)\right| d y, \\
& \bar{\rho}_{n}(x)=\int\left|\sum_{i=0}^{n} \Phi_{i}(x) \Phi_{i}(y)\right| d y,
\end{aligned}
$$

* Note the presence of the factor $\log ^{2} n$. From the mere convergence of $\sum_{n=2}^{\infty} c_{n}^{2}$ follows only (by the Riesz-Fischer theorem) the existence of a function $f(x)$ of class $L^{2}$ such that $c_{n}=\int f(x) \omega_{n}(x) d x,(n=0,1, \cdots)$.

$\dagger$ And, a fortiori, if $f(x)$ satisfies in $(a, b)$ a Lipschitz condition of an arbitrarily given order $\alpha(>0)$. 
where $x$ is given in $(a, b),(n=0,1, \cdots)$, and where $\rho_{n}=\max \rho_{n}(x), \bar{\rho}_{n}=\max \bar{\rho}_{n}(x)$ for $a \leqq x \leqq b$. The symbol $\max$ here and everywhere means, when necessary, upper bound. Hence, if $f(x)$ is bounded in $(a, b)$,

$$
\left|R_{n}(x ; f)\right| \leqq 2 M \rho_{n}(x) \leqq 2 M \rho_{n}, \quad(a \leqq x \leqq b ;|f(x)| \leqq M),
$$

by (11); and if $f(x)$ is continuous in the finite interval $(a, b)$,

$$
\left|R_{n}(x ; f)\right| \leqq E_{n}(f)\left\{1+\rho_{n}(x)\right\} \leqq E_{n}(f)\left(1+\rho_{n}\right)
$$

$(a \leqq x \leqq b)$, by $(12)$, where $G_{n}(x) \equiv \Pi_{n}(x ; f)$. Here $\rho_{n}(x)$ is the upper bound of $S_{n}(x ; f)$ for all $f(x)$ such that $|f(x)| \leqq 1$ in $(a, b)$. In fact, for such $f(x)$,

$$
\left|S_{n}(x ; f)\right| \leqq \int p(y)\left|K_{n}(x, y)\right| d y=\rho_{n}(x) .
$$

Moreover, this upper bound is actually attained by the function $f(y)=\operatorname{sgn} K_{n}(x, y)$.* Equation (53), by virtue of (32), shows at once that if $\left\{\rho_{n}(x)\right\}$ is bounded, then (6) converges to the value $f(x)$ at the given point $x$ for any continuous $f(x)$, the said convergence being uniform in any interval $c(a, b)$ where the Lebesgue constants remain uniformly bounded (in $x$ and $n$ ). On the other hand, if the sequence $\left\{\rho_{n}(x)\right\}$ is unbounded, it can be shown, following Haar [20], that there exist continuous functions whose expansions (6) diverge at the point $x$. In any case, if the order, with respect to $n$, of $\rho_{n}(x)$ or $\rho_{n}$ is known, equation (53), where $(a, b)$ is assumed to be finite, will show at a glance for what classes of continuous functions (6) converges, by means of (35). If, for example, $\rho_{n}(x)=O(\log n)$, then (6) converges to $f(x)$ at the given point $x$, provided $f(x)$ satisfies in $(a, b)$ a Dini-Lipschitz condition. In this connection it was shown by Rademacher [16] that

$$
\bar{\rho}_{n}(x)=O\left(n^{1 / 2}(\log n)^{3 / 2+\epsilon}\right)
$$

almost everywhere in $(a, b)$; hence, (43) converges to $(p(x))^{1 / 2} f(x)$ almost everywhere in $(a, b)$ if $f(x)$ satisfies in $(a, b)$ a Lipschitz condition of order $>1 / 2$.

* Note that $\operatorname{sgn} a=+1,-1,0$, corresponding to $a>0,<0,=0$. 
The above discussion clearly shows the importance of the so-called "zero-" or "kernel series" (série-noyau, Kogbetliantz) :*

$$
\sum_{n=0}^{\infty} \Phi_{n}(x) \Phi_{n}(y) \equiv(p(x) p(y))^{1 / 2} \sum_{n=0}^{\infty} \phi_{n}(x) \phi_{n}(y) .
$$

To illustrate, assume that

$$
\sum_{n=0}^{\infty} \phi_{n}(x) \phi_{n}(y)=0 \text { for } x \neq y, \quad(a \leqq c \leqq x, y \leqq d \leqq b),
$$

uniformly if $|x-y| \geqq \epsilon$. Assume further, in order to simplify the discussion and to bring out more clearly the underlying ideas, that (56) holds in $(a, b)$, $\dagger$ and let $f(x)$ be continuous at a certain interior point $x$ (where $p(x) \neq 0$ ). We have then $\ddagger$

$$
\begin{aligned}
S_{n}(x ; f) & =\left(\int_{a}^{x-\epsilon}+\int_{x-\epsilon}^{x+\epsilon}+\int_{x+\epsilon}^{b}\right) p(y) f(y) \sum_{i=0}^{n} \phi_{i}(x) \phi_{i}(y) d y \\
& \equiv i_{1}+i_{2}+i_{3},
\end{aligned}
$$

where $i_{1}=o(1), i_{3}=o(1)$, by $(56)$;

$$
\begin{aligned}
i_{2}=f(x) & \int_{x-\epsilon}^{x+\epsilon} p(y) \sum_{i=0}^{n} \phi_{i}(x) \phi_{i}(y) d y \\
& +\int_{x-\epsilon}^{x+\epsilon} p(y)[f(y)-f(x)] \sum_{i=0}^{n} \phi_{i}(x) \phi_{i}(y) d y=i_{2}{ }^{\prime}+i_{2}{ }^{\prime \prime} ;
\end{aligned}
$$

* A penetrating investigation of this and related zero-series by $\mathrm{E}$. Kogbetliantz for various classes of $O P$ has yielded in recent years extremely general results regarding convergence and summability of the expansion (6) (see, for example, [14]).

† Ordinarily (56) is satisfied when the series on the left is subjected to a certain process of summation, so that the conclusions drawn pertain not to ordinary convergence, but to summability. The latter, under additional hypotheses, may lead to ordinary convergence (see below, p. 72).

$\ddagger$ The end points need special attention. Thus, if $(a, b)$ is finite, we generally write $\int_{a}^{x-\epsilon}=\int_{a}^{a+\epsilon_{n}}+\int_{a+\epsilon_{n}}^{x-\epsilon}$, where $\epsilon_{n}=o(1)$ is properly chosen, and if $(a, b)$ is infinite, say $a=-\infty$, then we write $\int_{-\infty}^{-a}+\int_{-a}^{x-\epsilon}$, where $G(>0)$ is sufficiently large (or even depends on $n$ and increases indefinitely with $n$ ). Similar remarks apply to $b$. In other words, we must properly specify the behavior of $f(x)$ near the end points. 


$$
\begin{array}{r}
i_{2}^{\prime}=f(x)\left(\int_{a}^{b}-\int_{a}^{x-\epsilon}-\int_{x+\epsilon}^{b}\right) p(y) \sum_{i=0}^{n} \phi_{i}(x) \phi_{i}(y) d y \\
=f(x)+o(1) \quad \text { (by (8) and (56)); } \\
\left|i_{2}^{\prime \prime}\right| \leqq \eta(\epsilon)\left(\int_{a}^{b}+\int_{a}^{x-\epsilon}+\int_{x+\epsilon}^{b}\right) p(y)\left|\sum_{i=0}^{n} \phi_{i}(x) \phi_{i}(y)\right| d y \\
=\eta(\epsilon)\left(\rho_{n}(x)+o(1)\right) ; \\
(|f(y)-f(x)| \leqq \eta(\epsilon) \text { for } x-\epsilon \leqq y \leqq x+\epsilon ; \eta(\epsilon) \rightarrow 0, \text { as } \epsilon \rightarrow 0) .
\end{array}
$$

Thus, we again come across the Lebesgue constants, namely,

$$
S_{n}(x ; f)=f(x)+\eta_{1}(\epsilon) \rho_{n}(x)+o(1), \quad\left(\eta_{1}(\epsilon) \rightarrow 0, \text { as } \epsilon \rightarrow 0\right),
$$

and this shows that $\lim _{n \rightarrow \infty} S_{n}(x ; f)=f(x)$, if the sequence $\left\{\rho_{n}(x)\right\}$ is bounded, even though the continuity of $f(x)$ was here assumed at the point $x$ only.* The important point here is that, due to (56),

$$
\rho_{n}(x)=\int_{x-\epsilon}^{x+\epsilon} p(y)\left|\sum_{i=0}^{n} \phi_{i}(x) \phi_{i}(y)\right| d y+o(1), \quad(n \rightarrow \infty) .
$$

In general, the estimate of $\rho_{n}(x)$ requires the use of the asymptotic expression for $\phi_{n}(x)$. Another method based on a theorem of Fubini will be omitted [21].

4. Making Use of the Asymptotic Expression of $\phi_{n}(x)(n \rightarrow \infty)$. One can foresee that this method will prove the most powerful, for the said asymptotic expression is, we may say, a synthesis of the most intimate properties of $\phi_{n}(x)$. The essential features of this method may be exhibited as follows. For various classes of $O P$ the asymptotic expression of $\phi_{n}(x)$, for $n \rightarrow \infty$, has in the first approximation the following form:

$$
\phi_{n}(x)=A_{n}\left[\cos \left(n^{q} \psi+\omega_{n}\right)+\frac{B_{n}}{n^{p}}\right], \quad(q, p>0),
$$

and this holds in a certain subinterval of $(a, b)$, where $A_{n}, \psi$, $\omega_{n}, B_{n}$ are certain functions of $x$, with $\omega_{n}, A_{n}, B_{n}$ depending generally on $n$, and $A_{n}, B_{n}$ remaining bounded for $n \rightarrow \infty$. Thus, for example, for Legendre polynomials $[2,22]$ :

* In case $f(x)$ is of bounded variation in the neighborhood of the given point $x$ (the usual assumption made in dealing with (6)), the previous analysis, somewhat refined, would introduce the quantities $f(x \pm 0)$. 


$$
\begin{aligned}
& P_{n}(x)=\left(\frac{2}{2 n+1}\right)^{1 / 2} \phi_{n}(x, 1)=\frac{4}{\pi} \cdot \frac{2 \cdot 4 \cdots 2 n}{1 \cdot 3 \cdots(2 n+1)} \\
& \cdot \frac{1}{(2 \sin \phi)^{1 / 2}} \cdot\left\{\cos \left[\left(n+\frac{1}{2}\right) \phi-\frac{\pi}{4}\right]+\frac{1}{2} \cdot \frac{1}{2 n+3}\right. \\
&(60) \quad \frac{\cos \left[\left(n+\frac{3}{2}\right) \phi-\frac{3 \pi}{4}\right]}{2 \sin \phi}+\frac{1 \cdot 3}{2 \cdot 4} \cdot \frac{1 \cdot 3}{(2 n+3)(2 n+5)} \\
&\left.\cdot \frac{\cos \left[\left(n+\frac{5}{2}\right) \phi-\frac{5 \pi}{4}\right]}{(2 \sin \phi)^{2}}+\cdots\right\} \\
&=\left(\frac{2}{n \pi \sin \phi}\right)^{1 / 2}\left\{\cos \left[\left(n+\frac{1}{2}\right) \phi-\frac{\pi}{4}\right]+\frac{B_{n}(\phi)}{n}\right\}, \\
&(-1+\epsilon \leqq x=\cos \phi \leqq 1-\epsilon) .
\end{aligned}
$$

Similarly, for Hermite polynomials [13]:

$\phi_{n}(x)=e^{x^{2} / 2} \cdot \pi^{-1 / 2} \cdot\left(\frac{2}{n}\right)^{1 / 4} \cdot\left\{\cos \left(x(2 n)^{1 / 2}+\frac{n \pi}{2}\right)\right.$

$$
\left.+\left(\frac{x^{3}}{6}-\frac{x}{2}\right) \cdot(2 n)^{-1 / 2} \cdot \sin \left(x(2 n)^{1 / 2}+\frac{n \pi}{2}\right)+\frac{B_{n}(x)}{n}\right\},
$$

where $|x| \leqq A$ is finite and arbitrarily fixed.

In the first place, we find, by means of Darboux's formulas (9), an asymptotic expression for $K_{n}(x, y)$ and $K_{n}(x), x$ being a certain given point belonging to the interval where (59) is valid. Next, again rewrite (10) in the form

$$
\begin{aligned}
S_{n}(x ; f) & =\left(\int_{a}^{x-\epsilon}+\int_{x-\epsilon}^{x+\epsilon}+\int_{x+\epsilon}^{b}\right) p(y) f(y) K_{n}(x, y) d y \\
& \equiv i_{1}+i_{2}+i_{3}
\end{aligned}
$$

making the usual assumption that $f(x)$ is of bounded variation in the neighborhood of the point $x$. The most difficult part of the investigation, which requires great ingenuity (and of ten laborious computation) is to show that $i_{1}$ and $i_{3}$ tend to 0 , as $n$ tends to infinity, for the asymptotic expression (59) does not hold, as a rule, at the end points. Here we must specify the proper 
behavior of $f(x)$. The integrals $i_{1}$ and $i_{3}$ having been disposed of, we proceed to the relatively simple investigation of $i_{2}$. Using (59) and making a proper change of the variable of integration, we are led to a finite number of integrals of the form

$$
\begin{array}{lr}
\int_{\alpha}^{\beta} u(z) \sin _{\cos } m z d z, & (\alpha, \beta \text { finite or infinite }), \\
\int_{0}^{k} u(z) \frac{\sin m z}{\sin z} d z, & \text { Dirichlet integral }, \\
\int_{h}^{k} u(z) \frac{\sin m z}{\sin z} d z, & (0<h<k \leqq \pi / 2) .
\end{array}
$$

The behavior, for $m \rightarrow \infty$, of these integrals is well known from the theory of trigonometric series [4]. Thus, $(63)=o(1)$, if $|u(z)|$ is $L$-integrable in $(\alpha, \beta)$ (Riemann-Lebesgue theorem); $(64) \rightarrow(\pi / 2) u(+0)$, if $u(z)$ is of bounded variation in $(0, \pi / 2)$; $(65) \rightarrow o(1)$, for the same $u(z)$. These three limiting relations enable us to complete our investigation; (64) here furnishes the desired limit of $S_{n}(x ; f),(n \rightarrow \infty)$, in terms of $f(x \pm 0)$. The generality of the results obtained in this manner depends upon two factors: (i) the thoroughness of the study of the components $i_{1,3}$ in (62), by which we can avoid unnecessarily heavy restrictions to be imposed upon the differentiability and integrability properties of $f(x)$, also upon its behavior at the end points; (ii) the more or less comprehensive character of the asymptotic expression used, that is, its range of validity, order of magnitude, and nature of the remainder. Thus, Kogbetliantz [14] has obtained very general results on the convergence (and summability) of series according to Hermite or Laguerre polynomials, by using asymptotic expressions for $\phi_{n}(x)$ valid over an interval which increases indefinitely with $n$. Haar [23], making use of the second approximation in (59), was able to establish the most interesting result in the theory of Legendre polynomials - the so-called "equiconvergence theorem" (see Introduction): $f(x)$ being of the class $L^{2}$, its expansion (6) in series of Legendre polynomials behaves, with regard to convergence or divergence at any interior point $x=\cos \theta,(0<\theta<\pi)$, like the Fourier cosine-series expansion of $f(\cos \theta)$, that is, denoting by $\sigma_{n}(\theta)$ the partial sum of the latter series, we have 


$$
\lim _{n \rightarrow \infty}\left[S_{n}(\cos \theta)-\sigma_{n}(\theta)\right]=0, \quad(0<\theta<\pi) .
$$

Quite recently, Szegö [24] has developed for Jacobi polynomials with arbitrary $\alpha, \beta(>0)$ (see (5)) asymptotic expressions in terms of Bessel Functions, instead of trigonometric functions as in (59)-(61). His asymptotic expressions have the advantage of being valid uniformly in any left-hand neighborhood of the end point $x=1$. Thus one of the difficulties mentioned above is obviated, and we obtain in a simple manner the asymptotic expression of the Lebesgue constants and of many other definite integrals involving Jacobi polynomials. The most important application which Szegö makes of his asymptotic expressions is to derive a general "equiconvergence theorem," which, in case of Legendre polynomials, holds even for a more general class of functions than that in Haar's theorem (see below, p. 78).

In order to illustrate the difficulties arising in case of an infinite interval, we proceed to sketch the ingenious method of Uspensky in dealing with Hermite polynomials [13]. We write here

$$
\begin{aligned}
S_{n}(x ; f) & =\left(\int_{-\infty}^{-G}+\int_{-G}^{G}+\int_{G}^{\infty}\right) e^{-y^{2}} f(y) K_{n}(x, y) d y \\
& \equiv i_{1}+i_{2}+i_{3}, \quad(G>0, \text { sufficiently large }) .
\end{aligned}
$$

The integral $i_{2}$ is reduced to the Dirichlet integral as usual, and furnishes in the limit, for $n \rightarrow \infty,[f(x+0)+f(x-0)] / 2$, $(f(x)$ being of bounded variation in the neighborhood of the given point $x$ ). We now turn to $i_{3}$ (the analysis is similar for $i_{1}$ ). First, by Schwartz's inequality,

$$
i_{3}{ }^{2} \leqq \int_{G}^{\infty} e^{-y^{2}} f^{2}(y) d y \cdot \int_{G}^{\infty} e^{-y^{2} K_{n}{ }^{2}(x, y) d y .}
$$

The first factor in (66) does not cause trouble under the liberal assumption

$$
\int_{A}^{\infty} e^{-y^{2}} f^{2}(y) d y, \quad \text { and also } \int_{-\infty}^{-A} e^{-y^{2}} f^{2}(y) d y,
$$

exist for some $A>0$. 
We proceed to show that the second factor $\rightarrow 0$ as $n \rightarrow \infty$. By (9) and (10),

$$
\begin{aligned}
& \phi_{n+1}^{\prime}(x) \phi_{n}(x)-\phi_{n}^{\prime}(x) \phi_{n+1}(x)=\frac{(2 n+2)^{1 / 2}}{2} \int_{-\infty}^{\infty} e^{-y^{2} \beta_{n}{ }^{2}(y) d y} \\
& \equiv I_{n}=\left(\frac{2 n+2}{2}\right)^{1 / 2}\left(\int_{-\infty}^{-G}+\int_{-G}^{G}+\int_{G}^{\infty}\right) \\
& \equiv I_{n}^{\prime}+I_{n}^{\prime \prime}+I_{n}^{\prime \prime \prime} ; \\
& \beta_{n}(x, y)=\frac{\phi_{n+1}^{\prime}(x) \phi_{n}(y)-\phi_{n}^{\prime}(x) \phi_{n+1}(y)}{x-y}
\end{aligned}
$$

where $x$ is fixed and finite. The ingenious method of Uspensky consists in first evaluating asymptotically $I_{n}$, as a whole, using on the left side of (68) the asymptotic expressions of $\phi_{n}(x), \phi_{n}{ }^{\prime}(x)$ (the latter also being a Hermite polynomial), then evaluating asymptotically $I_{n}^{\prime \prime}$, by using the above asymptotic expressions under the integral sign (the interval of integration being finite). By subtraction we find at once the order of magnitude of $I_{n}^{\prime}, I_{n}^{\prime \prime \prime}$, and this completes the discussion of $i_{3}$, without any further restriction being imposed on $f(x)$. Laguerre polynomials are treated in a similar way. The criteria of convergence of (6) thus obtained are very sensitive indeed.

\section{Some Special Methods for Investigating the Convergence Properties of the Expansion (6).}

1. From Summability to Convergence. It is well known [25] that the Césaro $(C)$, Abel $(A)$, or Euler $(E)$ summability of an infinite series $\sum_{n=0}^{\infty} u_{n} \equiv U$ implies its ordinary convergence if $u_{n}$ is properly restricted. Thus, (i) if $U$ is $(C, k)$ summable, and $u_{n}=O(1 / n)$, then the series converges (Hardy); (ii) if $U$ is $A$-summable, and $u_{n}=O(1 / n)$, then the series converges (Littlewood). The existence of generating functions for the classical $O P$, that is,

$$
\Phi(x, t)=\sum_{n=0}^{\infty} c_{n} \phi_{n}(x) t^{n}, \quad\left(c_{n}=\text { const. }\right),
$$

whose explicit expressions are known, suggests in the first place Abel-summability. This was carried out by Hille [26] for Laguerre polynomials, where 


$$
\frac{e^{-x t}}{(1-t)^{\alpha}}=\sum_{n=0}^{\infty}(-1)^{n}\left(\frac{\Gamma(n+\alpha)}{\Gamma(n+1)}\right)^{1 / 2} \phi_{n}(x) t^{n},
$$

where $|t|<1$, and where $x$ is finite and arbitrary. Corresponding to the expansion (6), we construct the series

$$
F(x, t)=\sum_{n=0}^{\infty} f_{n} \phi_{n}(x) t^{n}, \quad(x \geqq 0, \text { fixed }) .
$$

Making use of the asymptotic expression for $\phi_{n}(x)$ and of the expression of $f_{n}$, we show, first, that $F(x, t)$, as a function of $t$, is holomorphic for $|t|<1$; next, that for $t \rightarrow 1-0, F(x, t) \rightarrow f\left(x_{0}\right)$ or $\left[f\left(x_{0}+0\right)+f\left(x_{0}-0\right)\right] / 2$, at every point $x_{0}$ of continuity or of discontinuity of the first kind, respectively; in other words, we show that (6) is $A$-summable, if $\int_{0}^{\infty} e^{-a x} x^{\alpha-1}|f(x)| d x$ exists for every $a>1 / 2$. By Littlewood's theorem, (6) will converge at $x=x_{0}$ (to the above value), if $f(x)$ is such that

$$
f_{n} \phi_{n}\left(x_{0}\right)=O\left(n^{-1}\right) \text {. }
$$

For $x_{0}>0$ and $\alpha=1, \phi_{n}\left(x_{0}\right)=O\left(n^{-1 / 4}\right)$; hence (71) is certainly satisfied if

$$
f_{n}=O\left(n^{-3 / 4}\right)
$$

which is shown to hold if $f(x)$ is subject to some further restrictions.

2. Application of the Closure Equation (15). We follow here, with considerable modification, the method developed by Stekloff [27] for the case where

$$
p(x) \geqq p_{0}>0,
$$

$p^{\prime}(x)$ exists and is bounded in the finite interval $(a, b)$,

$$
f^{\prime}(x) \text { is of class } L_{p}^{2} \text { in }(a, b) \text {. }
$$

Write

$$
f(x)=\sum_{i=0}^{n} f_{i} \phi_{i}(x)+R_{n} ; \quad f^{\prime}(x)=\sum_{i=0}^{n} f_{i} \phi_{i}^{\prime}(x)+R_{n}^{\prime},
$$

where $R_{n}$ denotes $R_{n}(x ; f)$, and where $R_{n}^{\prime}$ denotes $d R_{n} / d x$. By virtue of orthogonality, we get at once 


$$
\int p(x) R_{n} \phi_{i}(x) d x= \begin{cases}0, & (i \leqq n), \\ f_{i}, & (i>n) .\end{cases}
$$

This shows that

$$
\int p(x) R_{n} G_{n}(x) d x=0,
$$

and the second relation in (75) yields the fundamental relation

$$
\int p(x) R_{n} G_{1}(x) R_{n}^{\prime} d x=\int p(x) R_{n} G_{1}(x) f^{\prime}(x) d x,
$$

$\left(G_{1}(x)=g_{0}+g_{1} x ; g_{i}=\right.$ arbitrary constants, independent of $\left.n\right)$.

In (77) integrate by parts on the left and apply Schwartz's inequality on the right. This gives

$$
\begin{aligned}
&\left|\int p(x) R_{n} G_{1}(x) R_{n}{ }^{\prime} d x\right|=\mid \frac{1}{2}\left\{\left.p(x) R_{n}{ }^{2} G_{1}(x)\right|_{x=a} ^{x=b}\right. \\
&\left.-\int R_{n}{ }^{2} \frac{p^{\prime}(x)}{p(x)} p(x) G_{1}(x) d x-g_{1} \int p(x) R_{n}{ }^{2} d x\right\} \mid \\
& \leqq \int p(x) R_{n}^{2} d x \cdot \int p(x) f^{\prime 2}(x) G_{1}^{2}(x) d x=o(1), \\
&(n \rightarrow \infty ; \text { by (16)). }
\end{aligned}
$$

Moreover, by (73) and by use of the closure equation in its form (16),

$$
\int p(x) R_{n}^{2} \cdot \frac{p^{\prime}(x)}{p(x)} d x=o(1), \quad g_{1} \int p R_{n}^{2} d x=o(1), \quad(n \rightarrow \infty),
$$

so that (78) leads to

$$
\left.p(x) R_{n}^{2} G_{1}(x)\right|_{x=a} ^{x=b}=o(1), \quad(n \rightarrow \infty) .
$$

Taking here $G_{1}(x)=x-a, x-b$, we obtain

$$
p(b) R_{n}^{2}(b ; f)=o(1), \quad p(a) R_{n}^{2}(a ; f)=o(1), \quad(n \rightarrow \infty),
$$

which, expressed in words, states that, under conditions (73) and (74) the expansion (6) converges for $x=a, b$ to the values $f(a)$, $f(b)$, respectively.*

* In some cases this alone is a sufficient basis for the conclusion that (6) converges for all $x$ in $(a, b)$, 
In order to investigate $R_{n}$ at any interior point, we need a further assumption as follows:

$$
f^{\prime \prime}(x) \text { is of class } L_{p}^{2} \text {. }
$$

We then add to (75) the following relation:

$$
f^{\prime \prime}(x)=\sum_{i=0}^{n} f_{i} \phi_{i}^{\prime \prime}(x)+R_{n}^{\prime \prime}, \quad R_{n}^{\prime \prime}=\frac{d^{2}}{d x^{2}} R_{n},
$$

and proceeding as above, we get

$$
\begin{aligned}
& \int p(x) R_{n} G_{2}(x) R_{n}^{\prime \prime} d x=\int p(x) R_{n} G_{2}(x) f^{\prime \prime}(x) d x=o(1), \\
& (n \rightarrow \infty) \text {. }
\end{aligned}
$$

Now take $G_{2}(x)=(x-a)(b-x)$, which is $\geqq 0$ in $(a, b)$, and integrate by parts on the left side. Then

$$
\begin{aligned}
\int \frac{p^{\prime}(x)}{p(x)} & p(x) R_{n} R_{n}^{\prime} G_{2}(x) d x+\int p(x) R_{n} R_{n}^{\prime} G_{2}^{\prime}(x) d x \\
& +\int p(x) R_{n}^{\prime 2} G_{2}(x) d x=o(1), \quad(n \rightarrow \infty) .
\end{aligned}
$$

Here the middle term is $o(1)$, by (78), and

$$
\begin{gathered}
\left|\int \frac{p^{\prime}(x)}{p(x)} p(x) R_{n} R_{n}^{\prime} G_{2}(x) d x\right| \\
\leqq h \gamma\left(\int \frac{p^{\prime}(x)}{p(x)} R_{n}^{2} d x \cdot \int p(x) R_{n}^{\prime 2} d x\right)^{1 / 2}=h \gamma \cdot o(1)\left(I_{n}\right)^{1 / 2}, \\
h=\max \left|\frac{p^{\prime}(x)}{p(x)}\right|, \quad \gamma=\max \left|G_{2}(x)\right| \text { in }(a, b), \\
I_{n}=\int p(x) R_{n}^{\prime 2} G_{2}(x) d x,
\end{gathered}
$$

so that (82) becomes

$$
\left(I_{n}\right)^{1 / 2} \cdot o(1)+I_{n}=o(1), \quad(n \rightarrow \infty),
$$

whence the important result,

$$
I_{n}=\int p(x) R_{n}^{\prime 2}(x-a)(b-x) d x=o(1), \quad(n \rightarrow \infty)
$$


Consider now the identity

$$
\begin{aligned}
\left.p(x) \phi(x) R_{n}^{2}\right|_{a} ^{x}= & \int_{a}^{x}\left[p(x) \phi(x) R_{n}^{2}\right]^{\prime} d x=\int_{a}^{x} p^{\prime}(x) \phi(x) R_{n}^{2} d x \\
& +\int_{a}^{x} p(x) \phi^{\prime}(x) R_{n}^{2} d x+2 \int_{a}^{x} p(x) \phi(x) R_{n} R_{n}{ }^{2} d x \\
& \equiv i_{1}+i_{2}+i_{3}, \quad(\phi(x)=(x-a)(b-x)) .
\end{aligned}
$$

By the above considerations,

$$
\begin{aligned}
i_{1,2} & =o(1) \text { uniformly in } x, \quad(n \rightarrow \infty) ; \\
\left|i_{3}\right| & \leqq 2 \cdot\left(\frac{b-a}{2}\right)\left(\int p(x) R_{n}{ }^{2} d x \cdot \int p(x) R_{n}^{\prime 2} \phi(x) d x\right)^{1 / 2}=o(1),
\end{aligned}
$$

whence we reach the final conclusion that

$$
p(x)(x-a)(b-x) R_{n}^{2}(x)=o(1), \quad(n \rightarrow \infty),
$$

uniformly for $a \leqq x \leqq b$; that is, under conditions (73), (74) and $(80)$, the expansion (6) converges to $f(x)$ uniformly in the interval $(a+\epsilon, b-\epsilon)$.

The method is applicable to a more general $p(x)$, of the form $(x-a)^{\alpha-1}(b-x)^{\beta-1} q(x),(\alpha, \beta>0)$. We must point out, however, that the conditions imposed on $f(x)$ are far too stringent. This is compensated by the simplicity of the method, which requires neither the discussion of the coefficients $f_{n}$, nor the use of the asymptotic expression for $\phi_{n}(x)$. On the contrary, if we apply the above considerations to $f(x) \equiv \phi_{n+1}(x)$, we obtain directly

$$
\left|\phi_{n}(c)\right|=(2 n+1 / p(c))^{1 / 2}(1+O(1 / n)), \quad(c=a, b) .
$$

3. Use of the Differential Equations for the Classical OP. These can be written as follows:

$$
A(x) \phi_{n}^{\prime \prime}(x)+B(x) \phi_{n}^{\prime}(x)+C_{n} \phi_{n}(x)=0,
$$

where $A$ and $B$ are polynomials of degree $\leqq 2$, independent of $n$, and where $C_{n}$ denote constants.

$$
\begin{aligned}
& \text { (J) }\left(1-x^{2}\right) \phi_{n}{ }^{\prime \prime}(x)+[\alpha-\beta-(\alpha+\beta) x] \phi_{n}{ }^{\prime}(x) \\
& +n(n+\alpha+\beta-1) \phi_{n}(x)=0 \text {, } \\
& \text { (L) } x \phi_{n}^{\prime \prime}(x)+(\alpha-x) \phi_{n}^{\prime}(x)+n \phi_{n}(x)=0 \text {, } \\
& \text { (H) } \phi_{n}^{\prime \prime}(x)-2 x \phi_{n}^{\prime}(x)+2 n \phi_{n}(x)=0, \quad(n=0,1, \cdots) \text {. }
\end{aligned}
$$


From this we learn at once, by differentiation, that $\phi_{n}^{\prime}(x)$ is an $O P$ of the same class, with $p(x)$ replaced by $p_{1}(x)=A(x) p(x)$. Denoting the normalized $\phi_{n}^{\prime}(x)$ by $\psi_{n-1}(x)$, we derive, by a simple computation, the following important relation which holds true for all classical $O P$ :

$$
\phi_{n}^{\prime}(x)=\left(C_{n}\right)^{1 / 2} \psi_{n-1}(x), \quad(n=1,2, \cdots)
$$

where

$$
\int p_{1}(x) \psi_{m}(x) \psi_{n}(x) d x=\delta_{m n}
$$

where $m, n=0,1, \cdots$, and where $p_{1}(x)=A(x) p(x)$. Furthermore, it is readily seen that for all classical $O P$

$$
(A(x) p(x))^{\prime}=B(x) p(x), \quad A(x) p(x)=0 \text { for } x=a, b .
$$

Assuming now the existence in $(a, b)$ of $f^{\prime}(x)$ of the class $L_{p}^{2}$, (88) enables us to treat the coefficient $f_{n}$ as follows. We write

$$
\begin{aligned}
C_{n} f_{n} & =\int p(x) f(x) C_{n} \phi_{n}(x) d x \\
& =\int p(x) f(x)\left[-A \phi_{n}^{\prime \prime}(x)-B \phi_{n}^{\prime}(x)\right] d x,
\end{aligned}
$$

whence, by integration by parts, in virtue of (86) and (88),

$$
f_{n}=\frac{f_{n-1}^{\prime}}{\left(C_{n}\right)^{1 / 2}}
$$

where

$$
f_{n-1}^{\prime}=\int p_{1}(x) f^{\prime}(x) \psi_{n-1}(x) d x, \text { that is, } f^{\prime}(x) \sim \sum_{n=0}^{\infty} f_{n}{ }^{\prime} \psi_{n}(x) .
$$

We have now

$$
\left|f_{n} \phi_{n}(x)\right|=\left|f_{n-1}^{\prime} \cdot \frac{\phi_{n}(x)}{\left(C_{n}\right)^{1 / 2}}\right| \leqq \frac{1}{2}\left\{{f_{n-1}^{\prime 2}}^{2}+\frac{\phi_{n}^{2}(x)}{C_{n}}\right\} .
$$

Here the series $\sum_{n=1}^{\infty} f_{n-1}^{\prime 2}$ certainly converges (closure), and 
(J) $\quad \phi_{n}^{2}(x) / C_{n}=O\left(n^{-2}\right)$,

$$
(-1+\epsilon \leqq x \leqq 1-\epsilon)
$$

(L) $\quad \phi_{n}^{2}(x) / C_{n}=O\left(n^{-3 / 2}\right)$,

$$
(0<\epsilon \leqq x \leqq A ; A \text { finite, fixed arbitrarily); }
$$

$$
\phi_{n}{ }^{2}(x) / C_{n}=O\left(n^{-3 / 2}\right) \text {, }
$$$$
(|x| \leqq A) \text {. }
$$

Hence, the expansion (6) in series of classical OP converges uniformly and absolutely in the respective intervals as given in $(90)$, if $f^{\prime}(x)$ is of the class $L_{p}^{2}$ in $(a, b)$.

The same results could be obtained, without making use of the asymptotic expression for $\phi_{n}(x)$ (necessary to establish (90)), if we combine the use of the differential equation with that of closure as in the previous section(this would eliminate the introduction of $f^{\prime \prime}(x)$ ).

4. Comparison Method. The problem before us is: Given $p_{1}(x) \leqq p_{2}(x)$ in $(a, b)$, where $p_{1}(x)$ and $p_{2}(x)$ are two weightfunctions, find a corresponding qualitative relation between the partial sums of the two corresponding expansions (6) of the same function $f(x)$. This problem was solved by Szegö [28]. The basis of his analysis is the solution of the following two-parameter extremal problem.

Given in $(-1,1)$ a weight-function $p(x)$ and a function $F(x)$ such that $F(x) / p(x)$ and $F^{2}(x) / p(x)$ are both of class L, find max $\left\{\lambda G_{n}(\xi)+\mu \int_{-1}^{1} F(x) G_{n}(x) d x\right\}^{2},(\lambda, \mu$ parameters, $\xi$ a fixed point inside $(-1,1))$, for all $G_{n}(x)$ satisfying the condition $\int_{-1}^{1} p(x) G_{n}^{2}(x) d x=1$.

Introduce the $O P$ corresponding in $(-1,1)$ to $p(x)$ and write

$$
\begin{aligned}
G_{n}(x) & =\sum_{i=0}^{n} \gamma_{i} \phi_{i}(x), \gamma_{i} \text { being constants, with } \sum_{i=0}^{n} \gamma_{i}{ }^{2}=1 . \\
\frac{F(x)}{p} & \sim \sum_{i=0}^{\infty} \alpha_{i} \phi_{i}(x), \quad \alpha_{i}=\int_{-1}^{1} F(x) \phi_{i}(x) d x, \\
\frac{F(x)}{p(x)} & =\sum_{i=0}^{n} \alpha_{i} \phi_{i}(x)+R_{n} \equiv S_{n}\left(x ; \frac{F}{p}\right)+R_{n},
\end{aligned}
$$

$$
\begin{aligned}
U_{n}(\lambda, \mu) & \equiv\left\{\lambda G_{n}(\xi)+\mu \int_{-1}^{1} F(x) G_{n}(x) d x\right\}^{2} \\
& =\left\{\sum_{i=0}^{n} \gamma_{i}\left[\lambda \phi_{i}(\xi)+\mu \alpha_{i}\right]\right\}^{2}, \quad \sum_{i=0}^{n} \gamma_{i}{ }^{2}=1 .
\end{aligned}
$$


Then, by Cauchy's inequality and (13),

$$
\begin{aligned}
M_{n}(\lambda, \mu ; p) & \equiv \max U_{n}(\lambda, \mu)=\sum_{i=0}^{n}\left(\lambda \phi_{i}(\xi)+\mu \alpha_{i}\right)^{2} \\
M_{n}(\lambda, \mu ; p) & =\lambda^{2} K_{n}(\xi)+2 \lambda \mu \sum_{i=0}^{n} \alpha_{i} \phi_{i}(\xi)+\mu^{2} \sum_{i=0}^{n} \alpha_{i}{ }^{2} \\
& =\lambda^{2} K_{n}(\xi)+2 \lambda \mu S_{n}\left(\xi ; \frac{F}{p}\right)+\mu^{2} U_{n}\left(\frac{F}{p}\right) \\
U_{n}\left(\frac{F}{p}\right) & \equiv \sum_{i=0}^{n} \alpha_{i}{ }^{2} \leqq \int \frac{F^{2}(x)}{p(x)} d x \equiv U(p) .
\end{aligned}
$$

On the basis of the condition $\int_{-1}^{1} p(x) G_{n}{ }^{2}(x) d x=1$, we readily verify that

$$
\begin{gathered}
p_{1}(x) \leqq p(x) \leqq p_{2}(x) \text { in }(-1,1) \text { implies } \\
M_{n}\left(\lambda, \mu ; p_{2}\right) \leqq M_{n}(\lambda, \mu ; p) \leqq M_{n}\left(\lambda, \mu ; p_{1}\right) .
\end{gathered}
$$

Hence, the difference $M_{n}(\lambda, \mu ; p)-M_{n}\left(\lambda, \mu ; p_{2}\right)$, which is a quadratic form in $\lambda, \mu$, is positive for all real $\lambda, \mu$. Expressing the fact that its discriminant is non-positive, we get, replacing $F(x) / p(x)$ by $f(x)$, of class $L_{p}^{2}$, and writing $S_{n}(x ; p), K_{n}(x ; p)$, $U_{n}(p), \cdots$,

$$
\begin{aligned}
& p(x) \leqq p_{2}(x) \text { in }(-1,1) \text { implies } \\
& \begin{aligned}
{\left[S_{n}(\xi ; p)-S_{n}\left(\xi ; p_{2}\right)\right]^{2} } & \leqq U_{n}(p) \cdot\left[K_{n}(\xi ; p)-K_{n}\left(\xi ; p_{2}\right)\right] \\
& \leqq U(p)\left[K_{n}(\xi ; p)-K_{n}\left(\xi ; p_{2}\right)\right]
\end{aligned}
\end{aligned}
$$

where

$$
U(p)=\int p(x) f^{2}(x) d x
$$

This is the desired relation between $S_{n}(x ; p)$ and $S_{n}\left(x ; p_{2}\right)$. Incidentally we have found here before (94) two other important relations of the same kind:

$$
p_{1}(x) \leqq p(x) \leqq p_{2}(x) \text { in }(-1,1)
$$

implies

$$
K_{n}\left(\xi ; p_{1}\right) \leqq K_{n}(\xi ; p) \leqq K_{n}\left(\xi ; p_{2}\right), \quad U_{n}\left(p_{1}\right) \leqq U_{n}(p) \leqq U_{n}\left(p_{2}\right) .
$$


It now follows that

$$
p_{1}(x) \leqq p(x) \leqq p_{2}(x) \text { in }(-1,1)
$$

implies

$$
\left(S_{n}\left(\xi ; p_{1}\right)-S_{n}\left(\xi ; p_{2}\right)\right)^{2} \leqq U(p)\left[K_{n}\left(\xi ; p_{1}\right)-K_{n}\left(\xi ; p_{2}\right)\right] .
$$

Thus, if we succeed in choosing $p_{1}(x)$ and $p_{2}(x)$ in such a manner that

$$
\begin{aligned}
& p_{1}(x) \leqq p(x) \leqq p_{2}(x) \text { in }(-1,1) \\
& K_{n}\left(\xi ; p_{1}\right)-K_{n}\left(\xi ; p_{2}\right)=o(1),(n \rightarrow \infty ; \xi \text { given inside }(-1,1))
\end{aligned}
$$

then (94) leads directly to an equiconvergence theorem, namely,

$$
\lim _{n \rightarrow \infty}\left[S_{n}(\xi ; p)-S_{n}\left(\xi ; p_{2}\right)\right]=0
$$

Szegö shows that such a choice of $p_{1}(x)$ and $p_{2}(x)$ is possible, if

$$
\left\{\begin{array}{l}
p(x)=(1+x)^{\alpha-1}(1-x)^{\beta-1} q(x), \text { with } 1 / 2 \leqq \alpha, \beta \leqq 3 / 2 \\
q(x) \text { bounded and positive, } p(x) \text { is } R \text {-integrable in }(-1,1)
\end{array}\right.
$$

provided at the interior point $\xi$ under consideration $p(x)$ is continuous with its first and second derivatives. We then can take

$$
p_{1}(x)=\frac{\left(1-x^{2}\right)^{1 / 2}}{P_{1}(x)}, p_{2}(x)=\frac{1}{\left(1-x^{2}\right)^{1 / 2} P_{2}(x)},
$$

where $P_{1}(x)$ and $P_{2}(x)$ are polynomials greater than zero in $(-1,1)$; and we can choose the polynomials $P_{1}(x)$ and $P_{2}(x)$ so that, in addition to the first condition (97),

$$
\begin{aligned}
0 \leqq \int_{-1}^{1} \frac{\log p_{2}(x)-\log p(x)}{(x-\xi)^{2}} & \frac{d x}{\left(1-x^{2}\right)^{1 / 2}}<\epsilon ; \\
& \left(p_{1}(\xi)=p(\xi)=p_{2}(\xi)\right) .
\end{aligned}
$$

Szegö then obtains an explicit expression for $\phi_{n}\left(x ; p_{1,2}\right)$ (from a certain $n$ on) using Fejér's theorem on the trigonometric representation of positive polynomials [29], and by means of Darboux's formulas shows that the second condition (97) is also satisfied. Incidentally we find in this way an asymptotic expression for $K_{n}(x ; p)$ and $\phi_{n}(x ; p)$. Thus, $(98)$ is established. 
But this is an intermediate step only. The desired equiconvergence theorem in its final form is

$$
S_{n}(\xi ; p)-\frac{\sigma_{n}(\xi)}{p(\xi)\left(1-\xi^{2}\right)^{1 / 2}} \equiv o(1), \quad(n \rightarrow \infty),
$$

where $\sigma_{n}(\xi)$ represent the $n$th partial sum at the interior point $\xi=\cos \theta_{0}$ of the Fourier cosine-series expansion of the function $p(\cos \theta) f(\cos \theta)|\sin \theta|$. This is readily established, by first showing that (102) holds true for $p_{2}(x)$ (where the explicit expression of $\phi_{n}\left(x ; p_{2}\right)$ is known) and then again using (96). Taking $q(x) \equiv 1$, we obtain the equiconvergence theorem for Jacobi polynomials, with $1 / 2 \leqq \alpha, \beta \leqq 3 / 2$.

IV. Illustration of the General Methods Discussed Above by Means of Legendre Polynomials.

The Legendre polynomials

$$
\phi_{n}(x ; 1)=\left(\frac{2 n+1}{2}\right)^{1 / 2} P_{n}(x),
$$

where

$$
\frac{1}{\left(1-2 t x+t^{2}\right)^{1 / 2}}=\sum_{n=0}^{\infty} P_{n}(x) t^{n},
$$

have, among others, the following properties:

$\phi_{n}(-x) \equiv(-1)^{n} \phi_{n}(x) ;\left|\phi_{n}(x)\right| \leqq\left|\phi_{n}(+1)\right|=\left(\frac{2 n+1}{2}\right)^{1 / 2}$,

$(-1 \leqq x \leqq 1)$. Applying the preceding general methods to the expansion (6) of a given function $f(x)$ according to Legendre polynomials, we obtain the following results regarding its convergence.

(i) Case of continuous functions. From the estimate of $f_{n} \phi_{n}(x)$ we learn that (6) converges absolutely and uniformly in $(-1,1)$ if $f^{\prime}(x)$ satisfies therein a Lipschitz condition of order $>1 / 2$. From the estimate of $R_{n}(x ; f)$ we learn that (6) converges uniformly in any fixed interval $(-1+\epsilon, 1-\epsilon)$, if $f(x)$ satisfies in $(-1,1)$ a Dini-Lipschitz condition, and uniformly in the whole interval $(-1,1)$, if $f^{\prime}(x)$ is therein continuous.

(ii) Use of integral equations. The kernel here is [10] 


$$
K(x, y)=\left\{\begin{aligned}
\log (1-y)+\log (1+x)+ & 1-2 \log 2 \\
& (-1 \leqq y \leqq x \leqq 1) \\
\log (1+x)+\log (1-y)+1-2 \log 2 & \\
& (-1 \leqq x \leqq y \leqq 1) .
\end{aligned}\right.
$$

The series (6) converges absolutely and uniformly in $(-1,1)$ if $f(x), f^{\prime}(x)$, and $f^{\prime \prime}(x)$ are therein continuous.

(iii) Application of the Rademacher-Menchoff theorem. The series (6) converges almost everywhere in $(-1,1)$ if $f(x)$ is of bounded variation in $(-1,1)$, or if

$$
\begin{aligned}
\left|f\left(x^{\prime}\right)-f\left(x^{\prime \prime}\right)\right| \cdot|\log | x^{\prime}-x^{\prime \prime}||^{1+\epsilon} & <\text { const. } \\
& \left(-1 \leqq x^{\prime}, x^{\prime \prime} \leqq 1\right) .
\end{aligned}
$$

(iv) Use of Lebesgue constants. Here

$$
\rho_{n}(x) \sim \log n,(-1<x<1), \quad \rho_{n}( \pm 1) \sim n^{1 / 2} .
$$

The series $(6)$ converges uniformly in $(-1+\epsilon, 1-\epsilon)$ or in $(-1,1)$, if $f(x)$ satisfies in $(-1,1)$ a Dini-Lipschitz condition or a Lipschitz condition of order $>1 / 2$, respectively.

(v) Use of the asymptotic expression of $\phi_{n}(x)$. At any interior point $x=\cos \theta$, (6) behaves, regarding convergence or divergence, (a) like the ordinary Fourier series expansion of $f(\cos \theta)$, if $f(x)$ is of the class $L^{2}$ in $(-1,1)$; (b) like the ordinary Fourier series expansion of $f(\cos \theta)|\sin \theta|$, if $f(x)$ is of the class $L$ in $(-1,1)$ and $\int_{-1}^{1}\left(1-x^{2}\right)^{-1 / 4}|f(x)| d x$ exists.

We may add that if we wish to discuss the effect of singularities of $f(x)$ at the end points $x= \pm 1$ on the convergence (or summability) properties of (6), the zero-series (p. 65) proves the most effective tool.

We see that the weakest method is that of integral equations, the strongest one, that of asymptotic expressions. Note that the latter enter explicitly or implicitly in many other methods dealing with the convergence of (6). The above considerations apply not only to ordinary convergence, but also to summability of (6), as was pointed out above. They also remain valid in many parts if we replace $p(x) d x$ by the more general $d \psi(x)$, where $\psi(x)$ is monotonic non-decreasing in $(a, b)$, and use Stieltjes in tegrals. 
We have had to omit in our discussion many other interesting topics. The most important are those that follow.

(i) Gibbs' phenomenon, familiar from the theory of ordinary Fourier series.

(ii) The problem of Cantor. Can we have $\sum_{n=0}^{\infty} a_{n} \phi_{n}(x)=0$, ( $a \leqq x \leqq b)$, without having $a_{n}=0$ for all $n$ ? This is evidently equivalent to the problem of uniqueness of the expansion of a given $f(x)$ in series of orthogonal polynomials: can we have $\sum_{n=0}^{\infty} a_{n} \phi_{n}(x)=f(x), \sum_{n=0}^{\infty} b_{n} \phi_{n}(x)=f(x)$ for certain $x$, without having $a_{n}=b_{n}$ for all $n$ ?

(iii) The problem of Dubois-Reymond. Assume the existence of $\int p(x) f(x) \phi_{n}(x) d x \equiv f_{n}, \quad(n \geqq 0)$. If $\sum_{n=0}^{\infty} a_{n} \phi_{n}(x)=f(x)$ on a specified set $E \subset(a, b)$, can we conclude that $a_{n}=f_{n}$ for all $n$ ? This is equivalent to the problem of term by term integration of the above series.

These problems and many others on the general $O P$ offer a vast and fruitful field of research. In some special cases (polynomials of Legendre, symmetric Jacobi polynomials), interesting results have been obtained by Plancherel and Kogbetliantz.

\section{BIBLIOGRAPHY*}

1. J. Shohat (Jacques Chokhate), Théorie Générale des Polynomes Orthogonaux de Tchebicheff, Mémorial des Sciences Mathématiques, No. 66, 1934.

2. Darboux, Mémoire sur l'approximation des fonctions de très grands nombres, Journal de Mathématiques, (3), vol. 4 (1878), pp. 5-56, and pp. 377-416.

3. Correspondance d' Hermite et Stieltjes, 1905, vol. 2, letter 385.

4. E. W. Hobson, The Theory of Functions of a Real Variable, vol. 2, 2d ed., 1926.

5. M. Plancherel, Les problèmes de Cantor et de Dubois-Reymond dans la theorie des séries des polynomes de Legendre, Comptes Rendus, vol. 155 (1912), pp. 897-900.

6. Dunham Jackson, Series of orthogonal polynomials, Annals of Mathematics, (2), vol. 34 (1933), pp. 527-545.

7. Dunham Jackson, On the degree of convergence of a continuous function according to Legendre polynomials, Transactions of this Society, vol. 13 (1912), pp. 305-318.

8. J. Shohat, On the development of continuous functions in series of Tchebycheff polynomials, Transactions of this Society, vol. 27 (1925), pp. 537550.

9. J. Shohat, $A$ simple proof of a formula of Tchebycheff, Tôhoku Mathematical Journal, vol. 36 (1933), pp. 230-235.

* This list includes only the articles mentioned above. 
10. E. Goursat. Cours d' Analyse, vol. 3, 2d ed., 1915, pp. 539-542.

11. H. Weyl, Singuläre Integralgleichungen mit besonderer Berïcksichtigung des Fourierschen Integraltheorems. (Thesis, Göttingen, 1908; also Mathematische Annalen, vol. 66 (1909), pp. 273-324.)

12. Wera Lebedeff, Die Theorie der Integralgleichungen in Anwendung auf einige Reihenentwicklungen. (Thesis, Göttingen, 1906; also Mathematische Annalen, vol. 64 (1907), pp. 388-416.)

13. J. Uspensky, On the development of arbitrary functions in series of Hermite and Laguerre polynomials, Annals of Mathematics, (2), vol. 28 (1927), pp. 593-616.

14. E. Kogbetliantz, Recherches sur la sommabilité des séries d'Hermite, Annales de 1' École Normale Supérieure, (1932), pp. 137-221.

15. N. M. Kryloff, On the expansion in series of fundamental functions arising in the solution of a certain partial differential equation of the fourth order and on the expansion in series of Jacobi polynomials (Kieff, 1911, in Russian).

16. H. Rademacher, Reihen von allgemeinen orthogonalen Funktionen, Mathematische Annalen, vol. 87 (1922), pp. 112-138.

17. D. Menchoff, Fundamenta Mathematicae, vol. 4 (1923), pp. 82-105.

18. I. Natanson, On the convergence of series according to orthogonal polynomials, Comptes Rendus, Académie des Sciences URSS, (1934), pp. 209-212 (in Russian.)

19. A. Kolmogorov, ibid., (1933), pp. 291-294.

20. A. Haar, Zur Theorie der orthogonalen Funktionensysteme. (Thesis, Göttingen, 1909.)

21. Jacques Chokhate (J. Shohat), Sur la sommation de certaines séries des fonctions intégrables. Application aux fonctions orthogonales, Comptes Rendus, vol. 188 (1929), pp. 1475-1477.

22. E. W. Hobson, The Theory of Spherical and Elliptical Harmonics, 1931.

23. A. Haar, Mathematische Annalen, vol. 78 (1918), pp. 121-136.

24. G. Szegö, Asymptotische Entwicklungen der Jacobischen Polynome, Schriften der Königsberger Gelehrten Gesellschaft (Naturwissenschaftlichen Klasse), Heft 3 (1933).

25. K. Knopp, Theorie und Anwendungen der Unendlichen Reihen, $2 \mathrm{~d}$ ed., 1934.

26. Einar Hille, On Laguerre polynomials, First Note, Second Note, Third Note, Proceedings National Academy of Sciences, vol. 12 (1926), pp. 261-269, pp. 348-352.

27. W. Stekloff, Sur une application de la théorie de fermeture au problème $d u$ développement des fonctions arbitraries en séries procédant suivant les polynomes de Tchebycheff, Mémoires, Académie Impériale des Sciences de Petrograd, Classe Physico-Mathématique, vol. 33 (1914), pp. 1-58.

28. G. Szegö, Ueber die Entwickelung einer willkürlicher Funktion nach den Polynomen eines Orthogonalsystems, Mathematische Zeitschrift, vol. 12 (1922), pp. 61-94.

29. L. Fejér, Ueber trigonometrische Polynome, Journal für Mathematik, vol. 146 (1916), pp. 53-82.

The University of Pennsylvania 